\title{
A history of haematology: from Herodotus to HIV, Shaun R. McCann
}

\author{
Shaun R. McCann, ISBN: 9780198717607, Published: 03 March 2016. \\ Oxford University Press, 256 pages
}

\author{
M. F. McMullin ${ }^{1}$
}

Received: 24 April 2016/Accepted: 25 April 2016/Published online: 6 May 2016

(C) Royal Academy of Medicine in Ireland 2016

This medical history, one in a series aimed at clinical doctors but nonetheless readily accessible to a wider readership, traces the story of haematology through the ages. From the ancient Greeks surprisingly perceptive knowledge of the circulatory system through to the latest gene therapy, this fascinating study keeps the reader's attention at all times.

This scholarly work provides many intriguing insights from history. For example, the apparent appreciation of the genetics of haemophilia in the Talmud and the speculation of a link between disseminated intravascular coagulation and the attack of a vampire is remarkable.

Moving to the 20th century, the author gives a comprehensive and very entertaining account of blood transfusion, red cells, congenital haematological disorders, and infections. There are many revelations, but perhaps, the most thought-provoking to note is how far we have come in the last 30 years. This saw the rise of the spectre of HIV, its transmission to homosexual men, the ensuing epidemic, the great drives for a cure, and the discover of retro virals coming full circle to homosexual men returning as blood donors in 2013. This demonstrates, as always in haematology, that the laboratory, but especially, the bedside is supremely important in diagnosis.

The author moves on to review bone marrow transplantation and therapies for leukaemia. It is very noteworthy to review the developments that have occurred in one's own working lifetime (at least to those of us of a certain age). The revelation that the great pre-history hero of Irish mythology, Cuchulain, provided bone marrow to heal Cethern, son of Fintan, proves that there is nothing new under the sun.

The author then considers the different ways that haematology has developed in a smattering of countries reflecting the authors contacts and interests. However, this chapter is somewhat limited in its scope, and perhaps, should have cast its net wider to include other countries, such as Japan. He then gives us a comprehensive and shrewd evaluation of innovations in recent years. Finally, we have a careful exposition of the author's opinions in some matters and a robust defence of the arts of medicine.

The book throughout is exquisitely illustrated with great care in tracing and crediting suitable and enjoyable figures. The referencing is appropriate succinct and a very useful compendium. There are a few errors in areas such as having all genes in italics, but I am sure that these will be corrected in the future editions.

In summary, this is a fascinating and thoroughly readable history of blood, which will be of great interest to doctors, anyone who is involved in working with blood and those interested in the cycle of history. Professor McCann has produced a great book, which is highly recommended.

M. F. McMullin

M.McMullin@qub.ac.uk

1 Haematology, Belfast City Hosptial, Queen's University, Belfast, C Floor, Lisburn Road, Belfast BT 9 7AB, Northern Ireland, UK 